\title{
The past and future of delusions research: from the inexplicable to the treatable
}

\author{
P. A. Garety and D. Freeman
}

\section{Background}

Problems with the diagnosis and treatment of schizophrenia have led for a call to change strategy and focus on individual psychotic experiences. In recent years, research on delusions has led the way.

\section{Aims}

To update our 1999 review of almost 40 studies on delusions.

\section{Method}

A systematic literature search was conducted of reasoning and affective processes related to delusions.

\section{Results}

Over 200 studies were identified. The presence of 'jumping to conclusions' in individuals with delusions has been substantiated, the theory of mind account has not stood up to subsequent testing, and there is a promising new focus on the ways that affective processes contribute to delusional experience.

\section{Conclusions}

Theoretical work rendering delusions understandable can be translated into treatment; future clinical trials should focus on individual psychotic experiences as outcomes.

\section{Declaration of interest}

None.
Treatments for schizophrenia have reached a plateau. There has been no major breakthrough in the past decade. Whether we consider medication, psychological therapies or service level and social interventions, the period from 2000 to 2010 has been disappointing. Recent trials of antipsychotic medications demonstrated that they are of only moderate effect: many patients discontinue medication or show limited gains, the second-generation antipsychotics confer few if any clinical benefits over their first- generation counterparts, and many of these drugs have worrying effects on physical health. ${ }^{1}$ Firstgeneration psychological therapies, cognitive-behavioural therapy (CBT) and family interventions have also been shown to be of small to moderate effect, ${ }^{2}$ and are difficult to implement in practice, with widespread problems in training the workforce and ensuring effective models of delivery. Social interventions and psychiatric team configurations are no panacea either, and successful models in one setting, such as individual placement and support or assertive outreach, may not replicate their effects across time or culture. There are, nevertheless, some hopeful signs. Clozapine, although not new, is now clearly demonstrated to be a more effective medication than alternatives for people with unresponsive psychotic symptoms. ${ }^{3}$ Family interventions and CBT for psychosis are relatively new methods with considerable scope for improvement which have brought relief to many patients and carers. ${ }^{2}$ Early intervention and detection models confer benefits in engagement and early cost-effective treatment, ${ }^{4}$ whereas the recovery model reshapes professional efforts towards the personal values and goals of those using the service. However, few would disagree that we need to do much better.

One major problem may lie in the conceptualisation of schizophrenia as a single dichotomous category, underpinning most research. Genetic research has increasingly concluded that schizophrenia has overlap with other diagnoses, suggesting that it may lie on a spectrum of psychiatric disorders, better represented on a number of dimensions. ${ }^{5}$ In social psychiatry and epidemiology, convincing evidence has accumulated for psychosis-like experiences and traits in the general population, which share the same risk factors as those for the diagnosed disorder. ${ }^{6}$ This supports the long-standing idea that instead of conceptualising and researching schizophrenia in terms of a single disorder, qualitatively different from normality, we should think instead of quantitative traits across the whole population. Research also consistently demonstrates that the symptoms of schizophrenia load onto a number of relatively independent factors, including positive, negative, affective and disorganisation symptoms and also cognitive and/or motor dysfunctions. ${ }^{7}$ Within factors, there may be further reliable divisions. Thus, positive symptoms of hallucinations and delusions are consistently separated in these factor analyses, while there are subtypes of delusions, so that (for example) persecutory and grandiose delusions are distinct. ${ }^{8}$ The current controversies in the revision of the diagnostic systems for mental disorders, DSM-5 and ICD-11, echo many of these themes, with active debates about adopting multidimensional approaches, with major symptom or function domains, and clarifying the boundaries between psychosis and normality. ${ }^{9}$

\section{Delusions as a single symptom}

One solution, recommended by Owen et $a{ }^{10}$ is to 'focus on specific symptoms as well as cognitive and neurocognitive endophenotypes with the confounding effects of diagnostic practices removed' (p. 174). Individual symptom research is not a new idea. However, it has not been very much undertaken in psychosis. One exception is in the discipline of cognitive psychology, where the study of delusions as a single symptom has been actively pursued as a research strategy since the late 1980s. In 1999 we were able to review an emerging empirical cognitive psychology literature of delusions, consisting of 39 studies. ${ }^{11}$ This literature is now very substantially increased, with in excess of 200 further studies published since 1999. It is timely to revisit it.

The 1999 review provided an empirical basis for the subsequent development and elaboration of cognitive models of positive psychotic symptoms. ${ }^{11}$ These accept the importance of biological and social levels of explanation, but highlight the contribution of psychological mechanisms, involving both emotional and reasoning processes, to the development and 
persistence of psychosis. ${ }^{12}$ Thus, in the vulnerable individual, stress is held to trigger particular emotional and cognitive changes, resulting in anomalies of conscious experience such as hallucinatory voices. Both singly and in combination, specific reasoning and information processing biases, pre-existing schematic beliefs about the self and others, current emotional disturbance and social factors (e.g. isolation and adversity) are then considered to facilitate maladaptive appraisals of the origins of these anomalous mental states (Fig. 1). This may result in anomalous experiences becoming psychotic delusions and hallucinations. In this review we consider the current status of empirical support for the central proposition that disturbances in both reasoning and affective processes contribute to delusions.

\section{Method}

The list of studies included in our 1999 review paper was updated using systematic literature searches: see online Tables DS1-DS5.

\section{Results}

\section{Reasoning studies}

Cognitive models propose that processes that contribute to biased appraisals are causal factors in the development and persistence of delusions. Two reasoning biases have been repeatedly highlighted as candidates for making a causal contribution to delusions: jumping to conclusions and deficits in theory of mind. ${ }^{11}$

\section{Jumping to conclusions}

A tendency to use fewer data to reach a decision ('jumping to conclusions') is posited to contribute to delusion formation and persistence; thus, it is proposed that anomalous or ambiguous information is rapidly appraised and a delusional conclusion drawn on the basis of limited evidence, and without a thorough consideration of alternatives or a review of the evidence. Jumping to conclusions (JTC) has most often been assessed experimentally using a probabilistic reasoning task (the beads task). In a typical task, participants are shown jars with beads of two different colours in reverse proportions. Their task is to decide which of the two jars (now hidden from view) has been chosen, on the basis of beads drawn from only one jar. Commonly used proportions are 85:15 and 60:40. A number of measures can be taken. The most common variable employed is the number of items requested before making a decision ('draws to decision'); additionally, probability estimates, changes following potentially disconfirmatory evidence, confidence ratings and errors may be assessed. The JTC bias has been operationalised as when a decision is made after two or fewer beads on the $85: 15$ task. ${ }^{13}$

In 1999, reviewing 8 studies, we found that there was consistent evidence that individuals with delusions request less information before making their decision than psychiatric or non-clinical controls. A total of 61 further studies of the JTC bias have been published (online Tables DS1a-c). The clear majority of these studies and a meta-analysis have confirmed that JTC is characteristic of people with delusions, ${ }^{14}$ with about half showing the bias of gathering less evidence (i.e. deciding after two beads or fewer). For example, in a large, well-conducted study, Lincoln et al compared 71 patients with a diagnosis of schizophrenia with 68 participants from a non-clinical control group. ${ }^{15}$ The schizophrenia group was further divided into 44 people with acute delusions and 27 with a history of delusions who were in remission. They found that people with delusions took fewer draws to decision than the non-clinical control group and that participants with current delusions took fewer draws than those in remission. Draws to decision were associated with observer-rated delusions, suggesting that there is a specific association of JTC with this symptom, although this association became non-significant after controlling for IQ score and negative symptoms. Patients with delusions modified their responses with changing probabilities and feedback, but still made decisions relatively quickly even when decisions had negative consequences. The authors argue that this effect does not seem to be accounted for by impulsiveness, consistent with other studies. ${ }^{16}$ Among clinical studies that employed a design in which participants with

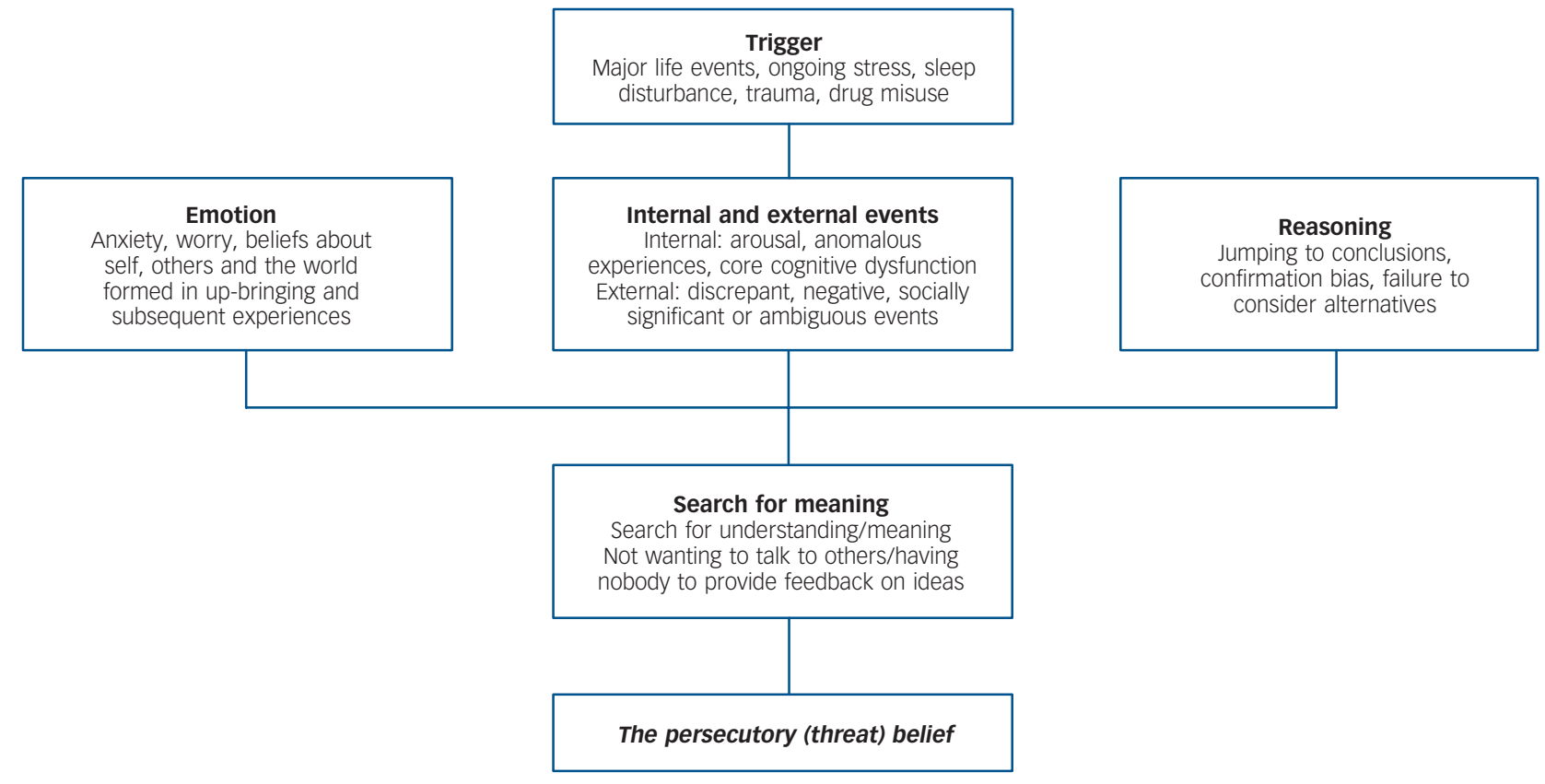

Fig. 1 Outline of factors involved in persecutory delusion development (from Freeman D, Pugh K, Antley A, Slater M, Bebbington P, Gittins M, et al. Virtual reality study of paranoid thinking in the general population. Br J Psychiatry 2008; 192: 258-63). 
delusions and a diagnosis of psychosis (mainly schizophrenia) were compared with a non-clinical control group on data-gathering, a large majority found JTC: there was a significant difference between the two groups, with the former showing reduced datagathering.

Many studies, like the Lincoln study cited, ${ }^{15}$ examined the relationship of JTC to delusions and not simply to a psychosis diagnosis. In general the larger studies showed evidence of a specific association of JTC with current delusional status, although there were some exceptions (online Table DS1a). Most of the nonclinical studies examined either people 'at risk' of delusions or people with 'delusion proneness' in the general population; the larger non-clinical studies also provided support for a specific association of JTC with delusional thinking or delusion proneness in the general population or in at-risk groups, although the JTC effect was attenuated in comparison with clinical groups (online Table DS1b). For example, Freeman et al found that a fifth of a sample of 200 healthy members of the general population showed a JTC bias; level of conviction in paranoid ideation was associated with the bias. ${ }^{17}$

We conclude that the JTC bias is associated with delusions in psychosis and is exacerbated in acute states, although remaining relatively stable in response to treatment in people with persistent symptoms. That it is also present in populations at risk or in remission, although in an attenuated form, suggests it is a trait representing liability to delusions. The cognitive mechanism of the bias is still unclear but there is emerging evidence to suggest that both anxiety and a working memory deficit may contribute to it. ${ }^{18,19}$ The work on JTC has also led to the more recent development of the concept of belief flexibility, that is, 'a metacognitive process of thinking about one's own delusional beliefs, changing them in the light of reflection and evidence and generating and considering alternatives' (p.374). ${ }^{13}$ Poor belief flexibility is common in people with delusions and related to, but distinct from, delusional conviction and JTC. ${ }^{20}$ Like JTC, it is unchanged by antipsychotic medication, but preliminary evidence suggests that JTC and belief flexibility may moderate change in delusions. ${ }^{20}$ Experimental tests indicate that JTC can be changed in the short term, and when combined with a brief intervention enhancing belief flexibility may lead to delusional change; these are early indications of the causal role of reasoning in delusions. ${ }^{21,22}$ Taken as a whole, the evidence suggests that it is likely that JTC and poor belief flexibility are relatively stable traits increasing susceptibility to the development of delusions, and may predict change over time. However, although both are associated with delusions, it has hitherto been little investigated whether JTC and belief flexibility differ by delusional subtype; recent indications suggest that they might. ${ }^{23}$

\section{Theory of mind}

Theory of mind (ToM) is defined as the ability to detect and reason about other people's mental states (e.g. beliefs, intentions). ${ }^{24}$ Frith was the first to propose that deficits in ToM might be a predisposing factor for persecutory delusions and delusions of reference. ${ }^{25}$ It has also been proposed that ToM is related to the disorganisation syndrome. ${ }^{26}$ Numerous studies have investigated ToM in patients with schizophrenia, finding their average performance to be below the performance of non-clinical control groups. Many have noted, however, that it has not been clearly established whether ToM deficits are related to persecutory or referential delusions, disorganisation or to schizophrenia in general (see, for example, the paper by Brune).$^{24}$ For this review we identified 43 additional studies of ToM, of which 38 were clinical studies (most typically with patients with a diagnosis of schizophrenia or with persecutory delusions, and 2 that employed 'high-risk' samples; details are given online in Table DS2a). A further three studies used a non-clinical sample and two examined participants with Asperger syndrome and delusions (online Tables DS2b,c). These studies used a wide variety of tasks, most involving brief vignettes and generally conceptualised as assessing either first-order ToM (the participant has to infer what another character is thinking) or second-order ToM (the participant is required to imagine what a character believes about the thoughts of someone else). Some tasks involved false beliefs, whereas others involved intentional deception.

The studies clearly confirmed that deficits in ToM were present in people with schizophrenia (a finding in nearly of all the studies that examined this), and deficits were somewhat inconsistently found to be present in people with persecutory delusions (the most frequently investigated specific symptom). However, there was little evidence that the deficit was specific to delusions or to persecutory delusions. The studies revealed a more consistent pattern of ToM deficits in association with negative symptoms than with positive symptoms or persecutory delusions. A recent meta-analysis cast further light on this: in 14 studies with over 600 patients no association was found between ToM and positive psychotic symptoms; but in contrast, employing data from 16 studies $(n=684)$ and 38 studies $(n=1869)$ respectively, significant moderate correlations were reported between ToM deficits and both disorganisation and negative symptoms. ${ }^{26}$ As this meta-analysis also demonstrated that ToM performance reliably correlated with neurocognition, the association with negative symptoms is to be expected. ${ }^{26}$ Non-clinical studies reviewed here also provide little support for an association of ToM deficits with delusions, including paranoia, and the recent studies of 'high-risk' groups are inconclusive (online Tables DS2a,b). We can conclude that ToM deficits are characteristic of schizophrenia, and although they are found in patients with delusions, they appear to be more strongly associated with negative and disorganisation syndromes rather than specifically with delusions.

\section{Emotion}

Traditionally, a sharp distinction has been drawn between emotional and psychotic problems. ${ }^{27,28}$ The dominant Jasperian perspective has been that the two problems were qualitatively different; diagnostically, psychotic symptoms 'trumped' signs of emotional problems. In contrast, the two main cognitive accounts of persecutory delusions place emotions central to their occurrence, albeit in different ways. ${ }^{29,30}$ Competing hypotheses for a number of years from the late 1990s concerned whether delusions defended against negative emotion or instead were a direct reflection of emotional concerns. It should be noted that this applies to persecutory delusions in particular, and there has been no comparable theoretical or empirical development for other delusion subtypes.

\section{Defences}

The most precise 'delusion as defence' model was put forward by Bentall et al. ${ }^{29}$ The central proposition is that individuals vulnerable to paranoia have low self-esteem of which they are unconscious, so that when negative events occur these events are explained by the hostility of others rather than allowing the negative thoughts about the self to reach consciousness. This theory has been tested in two main ways: first, by examining levels of self-esteem in paranoia, especially whether there are discrepancies between implicit and explicit self-esteem; and 
second, by assessing attributional style, especially whether external attributions are made for negative events. Testing overt self-esteem and attributional style is relatively straightforward, whereas examination of hypothetical manoeuvres around defences (i.e. discrepancies between conscious and unconscious processes) is more problematic methodologically.

There is a developing consensus that depression and overt low self-esteem, including negative ideas about the self, are associated with persecutory thinking. This can be seen very clearly in the overt self-esteem studies reviewed (online Tables DS3a-c). For instance, in a detailed study of patients with psychosis and a non-clinical control group, increasing levels of paranoia were associated with lower levels of self-esteem, ${ }^{31}$ and paranoid thinking was strongly associated with depressive cognitions in an epidemiological survey representative of the general population. ${ }^{32}$ In a large study comparing patients with paranoia and a non-clinical control group using structural equation modelling, an association of paranoia with a pessimistic thinking style (low self-esteem, pessimistic explanatory style and negative emotion) was reported. ${ }^{33}$ The evidence in our literature review clearly indicates that self-esteem is skewed towards the negative in people with paranoid thinking. Most, although by no means all, individuals with paranoia have lowered self-esteem. The presence of grandiosity may sometimes obscure this finding, since persecutory and grandiose delusions often co-occur, and it is seldom controlled for in studies. ${ }^{23}$ This general picture of low self-esteem is an important discovery that does not fit well with a defence account, in which relative preservation of mood and self-esteem might be expected. However, in Bentall et al's most recent revision of the model, failures of the defence mechanism are seen as occurring, and therefore fluctuations of self-esteem are instead seen as the critical variable in a dynamic system. ${ }^{34}$ Hence the theoretical models of paranoia have been converging.

Studies that try to compare overt and covert self-concepts use a variety of methods, perhaps reflective of the difficulties of operationalising a defence account. The sample sizes are typically small. In this review at least 11 out of 15 studies showed no discrepancy between overt and covert markers of self-esteem (online Table DS3b). The largest study compared 40 patients with current paranoid delusions, 25 patients with remitted delusions, 35 patients with depression and 36 non-clinical control participants on self-esteem and a memory recall task of positive and negative self-descriptive words designed to tap unconscious views of the self. ${ }^{35}$ No evidence was found of a discrepancy between overt and covert self-views in the delusion group. Indeed, evidence of defence mechanisms has not been obtained even for grandiose delusions, which are more obviously mood-enhancing beliefs.

The attributional defence account of persecutory delusions originated from studies of explicit judgements of the causes of events using the Attributional Style Questionnaire, ${ }^{36}$ which was developed to understand depression. An opposite pattern in individuals with delusions when compared with patients with depression was initially observed: taking the credit for positive events while externalising the cause of negative events. This selfserving style was thought to mask an underlying negative view of the self that was more in keeping with depression. The 44 studies identified here varied in the exact attributional style comparisons made, and whether associations with paranoia were considered across or within groups. We looked at internality ratings for negative and positive events separately (online Table DS4) and although not all studies presented data in this way, in general an internalising bias for positive events was not supported, whereas the evidence for and against externalising bias for negative events was equally balanced. Arguably the best method of approaching the issue might be to examine spontaneous utterances, and when this has been done it is normally found that patients with delusions make a greater number of external attributions for negative events. An externalising bias for negative events would plausibly be thought to occur in patients with paranoia; this is, of course, being expressed in the symptom experience. We suggest that such a style need not be tied to the further hypothesis of an unconscious low self-worth. Whether the externalising style is actually attributable to unassessed grandiosity in the delusion samples remains to be determined.

\section{Direct role of emotion}

An alternative hypothesis has emphasised that emotion - anxiety and depression in particular - has a direct (non-defensive) role in delusion formation and maintenance. ${ }^{30}$ Persecutory thoughts are considered an extension of anxious and depressive concerns about the person's own vulnerability and lack of worth (Fig. 2): anxiety provides the threat theme of persecutory delusions, worry elaborates the delusion content and keeps it in mind, while the use of safety behaviours leads to avoidance and the failure to process evidence inconsistent with the persecutory belief. The model was inspired by the frequent clinical observation of such emotional problems in patients. Both anxiety and depression have repeatedly been found to be common in people with persecutory thinking right across the spectrum of paranoia. Convincing experience sampling studies with patient groups have found that increases in anxiety predict the occurrence of paranoia, ${ }^{37}$ and there is initial evidence that experimentally increasing anxiety results in more paranoid thoughts. ${ }^{19}$ Anxiety has been found to be predictor of the subsequent development of psychosis. ${ }^{38}$ Consistent with these findings, psychotic-like experiences are more common in individuals with anxiety and depressive disorders. ${ }^{39}$

One psychological process used to explain the link between anxiety and paranoia has been a 'worry' thinking style. There is now reasonable evidence from ten studies of a role for worry in the development and persistence of paranoid thinking (online Table DS5). For example, in a longitudinal epidemiological

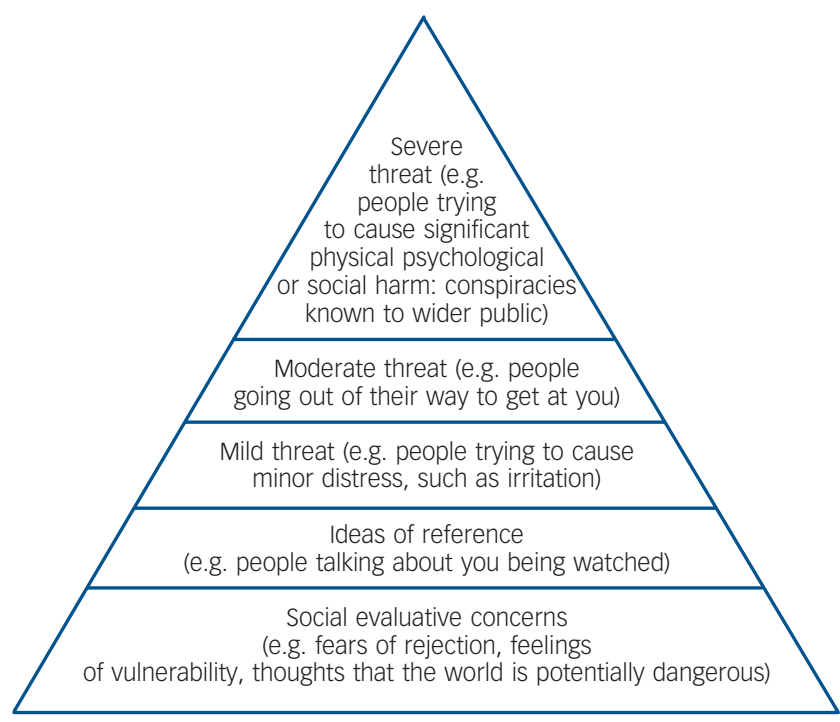

Fig. 2 The paranoia hierarchy (from Freeman D, Garety PA, Bebbington PE, Smith B, Rollinson R, Fowler D, et al. Psychological investigation of the structure of paranoia in a non-clinical population. Br J Psychiatry 2005; 186: 427-435. 
study levels of worry predicted both the onset and the persistence of paranoid thinking over 18 months, ${ }^{32}$ whereas worry and catastrophising have been found to predict the short-term persistence of persecutory delusions, ${ }^{40}$ as well as the occurrence of paranoia in experimental settings. ${ }^{41}$ Depression - and anxiety also - may arise from negative views about the self. It is proposed that paranoia builds directly on ideas that the self is vulnerable, may be rejected and is of lower social status. A study of a cohort of 300 patients with psychosis tested this hypothesis, with assessments at three time points over 1 year (online Table DS5). ${ }^{42}$ Depressed mood and negative ideas about the self predicted the strength of persecutory delusions over time, with only a weak effect in the reverse direction, and thus the predominant relationship is from affect to paranoia.

Positing a direct role for emotional processes in psychosis suggests that processes established as important in non-psychotic disorders may apply to delusions. Psychological processes underlying problems such as worry can be viewed as transdiagnostic maintenance factors. Over the past few years preliminary evidence has been found in individuals with persecutory thinking of the presence of plausible maintenance factors such as safety behaviours, an internal focus of attention, interpersonal sensitivity, experiential avoidance, sleep difficulties, emotion-focused coping strategies and negative visual images (online Table DS5). These processes now require more rigorous scrutiny in individuals with psychotic experiences. It is also likely that particular appraisals of psychotic experiences lead to negative affect, which in turn contributes to delusion persistence. ${ }^{27}$ Furthermore, appropriate weight also needs to be given to the study of factors that set emotional and psychotic experiences apart.

\section{Discussion}

The number of studies directly concerning delusions has increased considerably since our earlier review. In 1999 we reviewed 39 studies, and by the end of 2011 there were over 200 further studies to consider. What can be concluded from this research effort? The presence of biased 'jumping to conclusions' data-gathering reasoning processes in individuals with delusions has been substantiated. There is a promising new focus on emotional processes, which may influence many aspects of delusional experience. However, the review indicates that the theory of mind account has not stood up to subsequent testing. Although these studies, which take as their focus the explanation of delusions, have been on the periphery of the much greater research effort to explain schizophrenia, it is notable how many replicable findings are occurring, although the extent of publication bias is unknown. We take this as an indicator of the merits of adopting a single symptom approach. The previously inexplicable is now more explicable. Nevertheless, the study of delusions is still relatively new and there is considerable scope for development. It is striking how few studies provided adequate tests of causation. Longitudinal studies are rare, even the simple design of assessing predictors of outcome after acute admission. Experimental tests of the manipulation of key factors have been few in number and mainly confined to non-clinical groups. Measurement is an issue. Most studies have not used truly dimensional delusion assessments, and indeed these are not available for all delusion subtypes. Although the single symptom approach should not necessarily be constrained by diagnosis, it most frequently has been. There is little delusion research in clinical populations other than the non-affective psychosis diagnoses, but delusions are common in other diagnoses such as bipolar disorder and depression.
There are aspects of delusional experience receiving insufficient investigation. One of the earliest cognitive accounts of delusions emphasised how delusions are an attempt to explain anomalous internal experiences. ${ }^{43}$ In essence, odd experiences lead to odd ideas. This basic premise underlies the cognitive aspect of recent dopamine-related salience accounts of schizophrenia. ${ }^{44}$ There is a research challenge here in assessing cognitive anomalies such as salience, of which the individual with delusions has little awareness and for which new validated assessment methods are required. Yet misinterpretation of anomalous experiences and judgements based on such internal states are, in our view, crucial in the development of most delusions and hence require a concerted research effort. Furthermore, we see a close connection between affect and delusions, but the differential predictors have yet to be identified, ${ }^{41}$ and factors that distinguish the occurrence of different subtypes of delusions have yet to be systematically studied. ${ }^{23}$ Research also needs to go beyond the question of what causes a delusion. There may be rather separate answers to the questions of what leads to the initial emergence of delusional ideas, how strong belief convictions develop and dissipate, and what the causes are of significant distress and interference with functioning.

The research endeavour on delusions has been predominately from a cognitive perspective. A full account will need to link social and biological levels of explanation. Extensive work has identified the importance of social and environmental factors such as trauma, urbanicity, social deprivation, expressed emotion, migration and illicit drug use in the occurrence of psychosis. ${ }^{45}$ An explanation is needed of how these factors translate into biases in cognitive processing in delusions. Links of the delusion-related processes to biological correlates are rare, and there is an absence of a developmental literature on delusions even though it is established that psychotic-like experiences occur at a relatively young age. ${ }^{6}$ Our view is that genetic and biological work in psychosis will benefit from a better conceptualisation of the phenotype, that is focusing on separate quantitative traits, as has occurred for emotional disorders.

\section{Future treatments}

So what does the future hold for the treatment of psychosis? There is an important implication of this work for those working with psychosis in clinical services: the focus should be on understanding and treating the individual experiences that the patient is reporting, with the aim of reducing the distress and impact of each. In the clinic the experiences of the patient - rather than diagnosis should be placed centre stage. Specific interventions are therefore needed that target problems such as paranoia, grandiosity, hallucinations and anhedonia. The recent work on delusions also provides an illustration of how this can be taken a step further. If, for example, self-esteem, worry, insomnia, feelings of powerlessness, poor belief flexibility and jumping to conclusions are identified as causal factors for distressing delusions in an individual's case, then these become the actual targets for intervention. If the theoretical literature is correct, then improving each factor should reduce delusional experience. ${ }^{46}$ It is an explicit adoption of an interventionist-causal approach, ${ }^{47}$ to take one key causal factor at a time and target it in treatment. Recent psychological intervention studies for delusions provide encouraging results bearing this out, and are a significant step forward from first-generation CBT approaches. ${ }^{22,48}$ This approach, targeting identified mediators within a single symptom framework, has been successfully applied by Trower et al to the treatment of distress and behaviour in response to auditory hallucinations. ${ }^{49}$ Although our focus here is on mediators, the 
identification of moderators of treatment effects, such as the finding that people with carers responded better than those without to CBT for psychosis, ${ }^{50}$ also has the potential to inform the development of more effective treatments.

We have described the potential benefits of developing theoretically driven interventions for each distinct type of psychotic experience. Over the past decade in particular, delusions have become explicable in terms of cognitive processes, and this improved understanding is beginning to change psychological treatment. It is notable that in contrast to other psychological problems self-help has been little used in psychosis or application of technologies. Potentially synergistic effects of targeted cognitive interventions with short-term medication use have not been sufficiently tested. Treatment trials of both psychological and pharmacological interventions should look different. Rather than schizophrenia trials, we expect trials to have single psychotic experiences such as persecutory delusions or hallucinations as their focus. With these types of approaches we may well see greater therapeutic optimism in the treatment of psychotic experiences.

P. A. Garety, MA, MPhil, MA (Ed), PhD, FBPsS, Department of Psychology, Institute of Psychiatry, King's College London; D. Freeman, PhD, DClinPsy, CPsychol, FBPsS, Department of Psychiatry, University of Oxford, Warneford Hospital, Oxford, UK

Correspondence: Professor P. A. Garety, Box PO77, Institute of Psychiatry, King's College London, De Crespigny Park, London SE5 8AF, UK. Email: philippa.garety@kcl.ac.uk

First received 9 Aug 2012, final revision 25 Jan 2013, accepted 23 May 2013

\section{Funding}

D.F. is supported by a Medical Research Council Senior Clinical Fellowship. P.A.G. acknowledges support for clinical sessions from the National Institute for Health Research Biomedical Research Centre for Mental Health at the South London and Maudsley National Health Service Foundation Trust and Institute of Psychiatry, King's College London.

\section{References}

1 Jones $\mathrm{P}$, Barnes $\mathrm{T}$, Davies L, Dunn G, Lloyd H, Hayhurst K, et al. Randomized controlled trial of the effect on Quality of Life of second- vs first-generation antipsychotic drugs in schizophrenia. Arch Gen Psychiatry 2006; 63: 1079-87.

2 National Institute for Health and Clinical Excellence. Schizophrenia: Core Interventions in the Treatment and Management of Schizophrenia in Primary and Secondary Care (Update). NICE, 2009.

3 Lewis G, Barnes T, Davies L, Murray R, Dunn G, Hayhurst K, et al. Randomized controlled trial of effect of prescription of clozapine versus other second-generation antipsychotic drugs in resistant schizophrenia. Schizoph Bull 2006; 32: 715-23.

4 Mccrone $\mathrm{P}, \mathrm{Craig}$ TKJ, Power $\mathrm{P}$, Garety PA. Cost-effectiveness of an early intervention service for people with psychosis. Br J Psychiatry 2010; 196: 377-82.

5 Craddock N, Owen MJ. The Kraepelinian dichotomy - going, going . . . but still not gone. Br J Psychiatry 2010; 196: 92-5

6 Kelleher I, Keeley H, Corcoran P, Lynch F, Fitzpatrick C, Devlin N, et al. Clinicopathological significance of psychotic experiences in non-psychotic young people: evidence from four population-based studies. Br J Psychiatry 2012; 201: 26-32.

7 Peralta V, Cuesta MJ. Dimensional structure of psychotic symptoms: an itemlevel analysis of SAPS and SANS symptoms in psychotic disorders. Schizophr Res 1999; 38: 13-26.

8 Wigman JTW, Vollebergh WAM, Raaijmakers QAW, ledema J, van Dorsselaer S Ormel J, et al. The structure of the extended psychosis phenotype in early adolescence - a cross-sample replication. Schizophr Bull 2011; 37: 850-60.

9 van Os J. A salience dysregulation syndrome. Br J Psychiatry 2009; 194: 101-3.

10 Owen MJ, O'Donovan MC, Thapar A, Craddock N. Neurodevelopmental hypothesis of schizophrenia. Br J Psychiatry 2011; 198: 173-5.

11 Garety PA, Freeman D. Cognitive approaches to delusions: a critical review of theories and evidence. Br J Clin Psychol 1999; 38: 113-54.
12 Garety PA, Kuipers E, Fowler D, Freeman D, Bebbington PE. A cognitive model of the positive symptoms of psychosis. Psychol Med 2001; 31: 189-95.

13 Garety PA, Freeman D, Jolley S, Dunn G, Bebbington PE, Fowler D, et al. Reasoning, emotions and delusional conviction in psychosis. J Abnorm Psychol 2005; 114: 373-84.

14 Fine C, Gardner M, Craigie J, Gold I. Hopping, skipping or jumping to conclusions? Clarifying the role of the JTC bias in delusions. Cog Neuropsychiatry 2007; 12: 46-77.

15 Lincoln TM, Ziegler M, Mehl S, Rief W. The jumping to conclusions bias in delusions: specificity and changeability. J Abnorm Psychol 2010; 119: 40-9.

16 Lunt L, Bramham J, Morris RG, Bullock PR, Selway RP, Xenitidis K, et al. Prefrontal cortex dysfunction and 'Jumping to Conclusions': bias or deficit? J Neuropsychol 2012; 6: 65-78.

17 Freeman D, Pugh K, Garety P. Jumping to conclusions and paranoid ideation in the general population. Schizophr Res 2008; 102: 254-60.

18 Broome MR, Johns LC, Valli I, Woolley JB, Tabraham P, Brett C, et al. Delusion formation and reasoning biases in those at clinical high risk for psychosis. $\mathrm{Br} J$ Psychiatry 2007; 191 (suppl 51): s38-42.

19 Lincoln TM, Lange J, Burau J, Exner C, Moritz S. The effect of state anxiety on paranoid ideation and jumping to conclusions. An experimental investigation. Schizophr Bull 2010; 36: 1140-8.

20 So SH, Freeman D, Dunn G, Kapur S, Kuipers E, Bebbington P, et al. Jumping to conclusions, a lack of belief flexibility and delusional conviction in psychosis: a longitudinal investigation of the structure, frequency, and relatedness of reasoning biases. J Abnorm Psychol 2012; 121: 129-39.

21 Ross K, Freeman D, Dunn G, Garety P. A randomized experimental investigation of reasoning training for people with delusions. Schizophr Bull 2011; 37: 324-33

22 Waller H, Freeman D, Jolley S, Dunn G, Garety P. Targeting reasoning biases in delusions: a pilot study of the Maudsley Review Training Programme for individuals with persistent, high conviction delusions. Behav Ther Exp Psychiatry 2011; 42: 414-21.

23 Garety P, Gittins M, Jolley S, Bebbington P, Dunn G, Kuipers E, et al Differences in cognitive and emotional processes between persecutory and grandiose delusions. Schizophr Bull 2012; 39: 629-39.

24 Brune M. 'Theory of Mind' in schizophrenia. Schizophr Bull 2005; 31: 21-42. 25 Frith CD. The Cognitive Neuropsychology of Schizophrenia. Erlbaum, 1992.

26 Ventura J, Wood RC, Hellemann GS. Symptom domains and neurocognitive functioning can help differentiate social cognitive processes in schizophrenia: a meta-analysis. Schizophr Bull 2011; 39: 102-11.

27 Birchwood M. Pathways to emotional dysfunction in first-episode psychosis Br J Psychiatry 2003; 182: 373-5.

28 Freeman D, Garety PA. Connecting neurosis and psychosis: the direct influence of emotion on delusions and hallucinations. Behav Res Ther 2003; 41: 923-47.

29 Bentall RP, Kinderman P, Kaney S. The self, attributional processes and abnormal beliefs: towards a model of persecutory delusions. Behav Res Ther 1994; 32: 331-41.

30 Freeman D, Garety PA, Kuipers E, Fowler D, Bebbington PE. A cognitive model of persecutory delusions. Br J Clin Psychol 2002; 41: 331-47.

31 Thewissen V, Bentall R, Lecomte T, van Os J, Myin-Germeys I. Fluctuations in self-esteem in paranoia in the context of daily life. J Abnorm Psychol 2008; 117: 143-53.

32 Freeman $\mathrm{D}$, Stahl $\mathrm{D}$, McManus $\mathrm{S}$, Meltzer $\mathrm{H}$, Brugha $\mathrm{T}$, Wiles $\mathrm{N}$, et al. Insomnia, worry, anxiety and depression as predictors of the occurrence and the persistence of persecutory ideation. Soc Psychiatry Psychiatr Epidemiol 2012; 47: 1195-203.

33 Bentall RP, Rowse G, Shryane N, Kinderman P, Howard R, Blackwood N, et al. The cognitive and affective structure of paranoid delusions: a transdiagnostic investigation of patients with schizophrenia spectrum disorders and depression. Arch Gen Psychiatry 2009; 66: 236-47.

34 Bentall RP, Corcoran R, Howard R, Blackwood N, Kinderman P. Persecutory delusions: a review and theoretical interpretation. Clin Psychol Rev 2001; 21: 1143-92.

35 Vazquez C, Diez-Alegria C, Hernandez-Lloreda MJ, Moreno MN. Implicit and explicit self-schema in active deluded, remitted deluded, and depressed patients. Behav Ther Exp Psychiatry 2008; 39: 587-99.

36 Peterson C, Semmel A, Von Baeyer C, Abramson L, Metalsky GI, Seligman MEP. The Attributional Style Questionnaire. Cognitive Ther Res 1982; 3: 287-300

37 Ben-Zeev D, Morris S, Swendson J, Granholm E. Predicting the occurrence, conviction, distress and disruption of different delusional experiences in the daily life of people with schizophrenia. Schizophr Bull 2012; 38: 826-37. 
38 Jones $\mathrm{P}$, Rodgers $\mathrm{B}$, Murray $\mathrm{R}$, Marmot $\mathrm{M}$. Child developmental risk factors for adult schizophrenia in the British 1946 birth cohort. Lancet 1994; 344: 1398-402.

39 Varghese D, Scott J, Welham J, Bor W, Najman J, O'Callaghan M, et al. Psychotic-like experiences in major depression and anxiety disorders. Schizophr Bull 2011; 37: 389-93.

40 Startup H, Freeman D, Garety PA. Persecutory delusions and catastrophic worry in psychosis: developing the understanding of delusion distress and persistence. Behav Res Ther 2007; 45: 523-37.

41 Freeman D, Gittins M, Pugh K, Antley A, Slater M, Dunn G. What makes one person paranoid and another person anxious? The differential prediction of social anxiety and persecutory ideation in an experimental situation. Psycho Med 2008; 38: 1121-32.

42 Fowler D, Hodgekins J, Garety P, Freeman D, Kuipers E, Dunn G, et al. Negative cognition, depressed mood, and paranoia: a longitudinal pathway analysis using structural equation modeling. Schizophr Bull 2012; 38 1063-73.

43 Maher BA. Delusional thinking and perceptual disorder. $J$ Individ Psychol 1974; 30: 98-113.
44 Howes O, Kapur S. The dopamine hypothesis of schizophrenia. Schizophr Bull 2009; 35: 549-62.

45 Van Os J, Kenis G, Rutten BPF. The environment and schizophrenia Nature 2010; 468: 203-12.

46 Freeman D. Improving cognitive treatments for delusions. Schizophr Res 2011; 132: 135-9.

47 Kendler KS, Campbell J. Interventionist causal models in psychiatry. Psychol Med 2009; 39: 881-7.

48 Foster C, Startup H, Potts L, Freeman D. A randomised controlled trial of a worry intervention for individuals with persistent persecutory delusions. Behav Ther Exp Psychiatry 2010; 41: 45-51.

49 Trower P, Birchwood M, Meaden A, Byrne S, Nelson A, Ross K. Cognitive therapy for command hallucinations: randomised controlled trial. Br J Psychiatry 2004; 184: 312-20.

50 Garety PA, Fowler DG, Freeman D, Bebbington P, Dunn G, Kuipers E. Cognitive-behavioural therapy and family intervention for relapse prevention and symptom reduction in psychosis: randomised controlled trial. Br J Psychiatry 2008; 192: 412-23.

Suicide may be influenced by a wide range of social and economic factors, including adverse economic circumstances, social fragmentation and isolation, and cultural attitudes towards suicide. Psychiatric disorders, especially depression, bipolar disorder, schizophrenia and alcohol misuse, are present in most individuals dying by suicide. Key psychological factors are a sense of entrapment with no escape, hopelessness, shame and guilt. Risk can be increased by exposure to suicidal behaviour of others via social networks or the media (especially where reporting is dramatic and includes details of methods of suicide). Strong religious beliefs, having a partner and children may offer some protection. 